\title{
Radiological study of fabella in Omani subjects at a tertiary care center
}

\author{
Omar Darwish Al Matroushi ${ }^{1}$, Srinivasa Rao Sirasanagandla ${ }^{2}$, Ammar Al Shabibi $^{1}$, \\ Athari Al Obaidani ${ }^{3}$, Humoud Al Dhuhli ${ }^{4}$, Sanjay Jaju ${ }^{5}$, Mohamed Al Mushaiqri ${ }^{2}$ \\ ${ }^{1}$ College of Medicine and Health Sciences, Sultan Qaboos University, Muscat, ${ }^{2}$ Department of Human and Clinical Anatomy, College of Medicine \\ and Health Sciences, Sultan Qaboos University, Muscat, ${ }^{3}$ Radiology Residency Program, Oman Medical Specialty Board, Muscat, ${ }^{4}$ Department of \\ Radiology and Molecular Imaging, College of Medicine and Health Sciences, Sultan Qaboos University, Muscat, ${ }^{5}$ Department of Family Medicine \& \\ Public Health, College of Medicine and Health Sciences, Sultan Qaboos University, Muscat, Oman
}

\begin{abstract}
Ethnic diversity is associated with variability in the prevalence rates of fabella. We aimed to evaluate the prevalence and the radiological features of fabella in Omani patients. This is a retrospective analysis of hospital electronic database of patients referred for radiological investigations (radiographs and magnetic resonance imaging) of the knee, at a tertiary care referral center. Descriptive statistics were performed to determine the prevalence of fabella. Chi-square test was used to determine the association between sex or age with respect to the presence of fabella. A total of 813 knee radiographs were reviewed for the presence of fabella. Fabella was found in $24.1 \%$ of total cases. A statistically significant sex difference was observed with respect to the presence of fabella in left knees in males $(P<0.01)$. The presence of fabella was significantly associated with age groups for the right $(P<0.05)$ and left knees $(P<0.01)$. In magnetic resonance imaging film reviews, all the identified fabellae (20.2\%) were bony structures and were located within the lateral head of the gastrocnemius muscle. There were no cartilaginous fabellae detected. The current study revealed a prevalence of $24.1 \%$ of fabella in Omani subjects which is almost similar to the results as seen in Caucasian ethnic populations.
\end{abstract}

Key words: Prevalence, Sesamoid bones, Knee joint, Radiography

Received July 24, 2020; 1st Revised December 10, 2020; 2nd Revised April 7, 2021; Accepted April 7, 2021

\section{Introduction}

Fabella is one of the sesamoid bones in the lower limb. The term fabella refers to a "little bean" in Latin [1]. The fabella is located in the posterolateral corner of the knee on the lateral femoral condyle and embedded within the tendon of the lateral head of the gastrocnemius muscle. It can be felt by palpation and it may articulate with the posterior aspect of the lateral femoral condyle [2-5]. The fabella is formed

\section{Corresponding author:}

Srinivasa Rao Sirasanagandla (1)

Department of Human and Clinical Anatomy, College of Medicine and Health Sciences, Sultan Oaboos University, Muscat 123, Oman

E-mail: srinivasa@squ.edu.om by endochondral ossification, where it appears initially as a cartilaginous nodule and subsequently converts into a bone. However, in few individuals it remains unossified and persists as a cartilaginous nodule [6]. The main function of this bone is to stabilize the posterolateral structures of the knee joint through the fabellofibular ligament [7]. The fabella is variable in size and only a few studies have documented its dimensions. Its size changes from a tiny dot to a large body with a width of $22 \mathrm{~mm}$ [8]. It has been reported that the larger the size of the fabella, the greater the chances of it developing the symptoms [9].

Clinical identification of fabella is easy and frequently detected in the lateral knee radiographs. Rarely, in conditions such as osteoarthritic knees and intra-meniscal calcifications, this bone is confused with the intra-articular loose bodies or osteophytes $[6,10]$. Although it is seen continuous- 
ly in the tendon of gastrocnemius muscle in the non-hominoid animals like cats and dogs $[6,11,12]$, in humans it is considered as an anatomical variant. Epidemiological studies have confirmed the ethnic variability in the prevalence rates of fabella among different populations. The reported prevalence rate of fabella ranged from $8.7 \%$ to $31.3 \%$ in western population $[6,11,13], 31.25 \%$ to $85.8 \%$ in Asian population $[5,14,15]$. Sexual dimorphism in the variations of fabella has been reported with a male dominance [16]. The documented prevalence of fabella ranged from $4.3 \%$ to $52.8 \%$ in males [17] and 3.3\% to 50\% in females [18]. Both genetic and environmental factors influence have been demonstrated in variations of fabella. Evidence suggests that currently fabella occurrence is around 3.5 times more than seen 100 years ago indicating that a variation in prevalence is also of evolutionary significance [16].

The fabella is known to be associated with some clinical
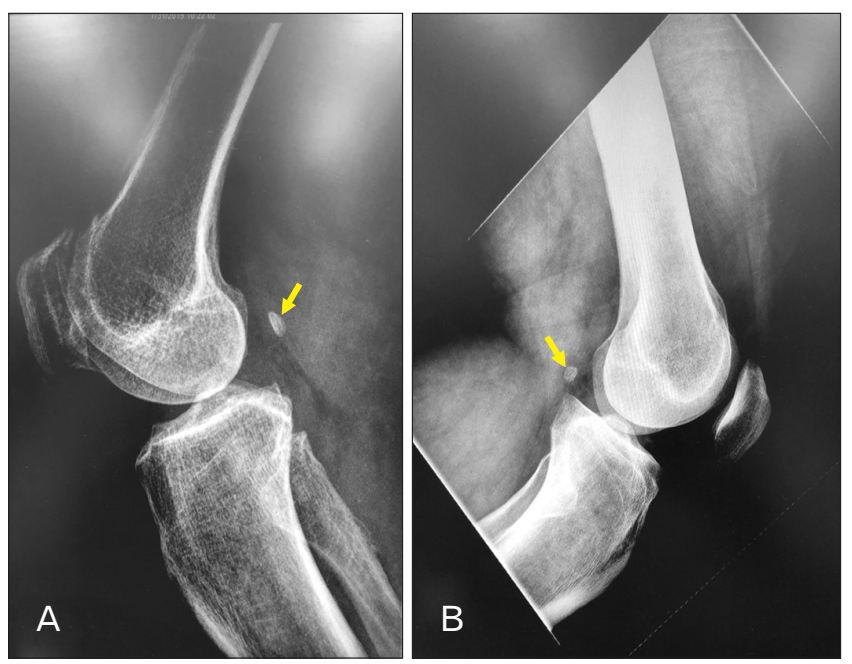

Fig. 1. Lateral view of right (A) and left (B) knee radiograms showing the presence of bony fabella (arrows) in two different Omani subjects. conditions, disorders and diseases. Some of these include osteoarthritis, chondromalacia and rheumatoid arthritis $[19,20]$. It may compress the popliteal artery and the peroneal neve and causes popliteal artery entrapment syndrome and common peroneal nerve neuropathy [21-23], respectively. Furthermore, the fabella itself can be involved in dislocations and fractures resulting in fabellar syndrome [24-27]. However, the baseline information on the prevalence and radiological characteristics of the fabella among the Omani population remains unexplored.

\section{Materials and Methods}

This is a retrospective analysis of the Electronic Medical Records database of adult Omani patients (aged $\geq 20$ years) at a tertiary care referral center in the Department of Radiology and Molecular Imaging in Oman from January 2019 to December 2019. The patients who were referred for radiological investigations of the knees i.e., radiographs (for prevalence and associated factors) and magnetic resonance imaging (MRI) (for tissue type only) in the year 2019 were included in the study. The Picture Archiving and Communication System (PACS) (Philips IntelliSpace version 4.4.516.21, Foster City, CA, USA) was used to review both the radiographs and MRI films. Lateral radiographs of the knee were used to identify the presence or absence of the bony fabella (Fig. 1). The tissue type of fabellae were documented exclusively based on the MRI findings on different planes of the knee (sagittal view/axial view) (Fig. 2). For MRI review, patients more than 45 years were excluded as increasing age affects the fabellar dimensions and causes ectopic calcification. Patients with a history of knee fractures and injuries, ligament injuries, areas of intra-articular loose bodies along the nonOmani patients were excluded. Institutional ethical com-

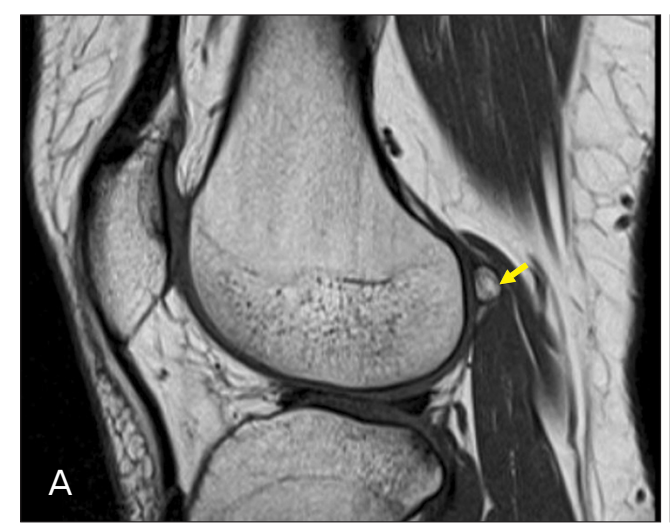

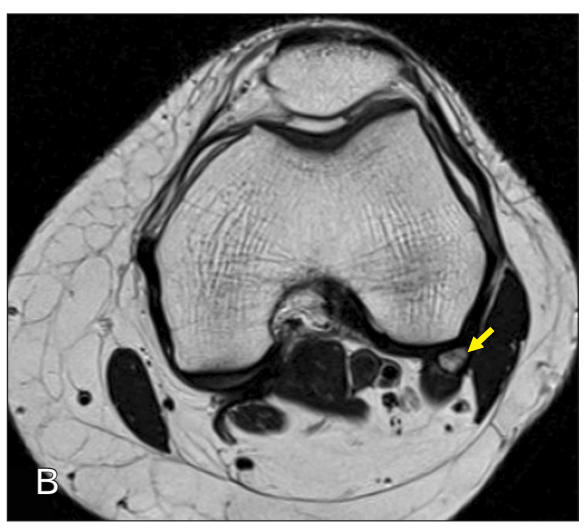

Fig. 2. Sagittal (A) and axial (B) magnetic resonance imaging planes showing the presence of bony fabella (arrows). 
mittee approval was obtained prior to the start of the study (SQU-EC/209/19). Waiver of written informed consent was also obtained.

\section{Data analysis}

The statistical analysis was performed by IBM SPSS software package (ver. 23; IBM Corp., Armonk, NY, USA) for windows. Descriptive statistics were used for analysis. Chi-square test was used to determine the association between sex or age with respect to the presence of fabella. A Pvalue $<0.05$ was considered statistically significant.

\section{Results}

\section{Radiographic films review}

A total of 813 knee radiographs were reviewed. Among these, 388 (47.7\%) were males and 425 (52.3\%) were females and their mean age was 56.9 years (SD: 20.6 years; range: 20-100 years). The presence of a fabella was noted in 196 knees indicating an overall prevalence of $24.1 \%$, in this study group. The sex-wise distribution of the fabella is presented in Table 1. The fabella was identified in 111 males $(28.6 \%$ of total males radiographs, $13.7 \%$ of total radiographs) and in 85 females (20.0\% of 425 females radiographs, $10.5 \%$ of the total radiographs). A statistical significant sex difference was observed in the occurrence of fabella in the left knee $(P<0.01)$. However, no statistically significant sex difference was observed in the right knee $(P=0.09)$. The distribution of the fa- bella according to different age groups is presented in Table 2 . The presence of fabella is significantly associated with age groups for the right $(P<0.05)$ and left knees $(P<0.01)$ indicating that the ossification of fabella increases with an increasing age.

\section{MRI knee review}

A total of 119 MRI films of the knee were examined to identify the presence of fabella. The presence of fabella was noted in $20.2 \%(24 / 119)$ of cases. All the fabellae identified were bony structures and located within the lateral head of the gastrocnemius muscle. There were no cartilaginous fabellae detected in the study group.

\section{Discussion}

Evidence from the epidemiological studies conducted in various parts of the world have indicated that the prevalence rate of fabella varies greatly among different ethnic groups (Table 3) $[5,6,8,10-15,28-34]$. All these studies have used either plain radiographs, MRI, cadaver dissection, or combinations of at least two methods to identify the fabella. Prevalence rate of fabella is found to be most common in Asian populations, followed by those from Oceania, South America, Europe, the Middle East, North America, and Africa [2]. Most of the studies from Far East Asian countries were conducted on cadavers in which cartilaginous fabella can easily be identified, which may have contributed to high prevalence $[5,8,11,12,14,30,31]$. Using the data from the

Table 1. Association of presence of fabella with respect to sex

\begin{tabular}{|c|c|c|c|c|c|}
\hline \multirow{2}{*}{ Sex } & \multicolumn{2}{|c|}{ Right knee } & \multicolumn{2}{|c|}{ Left knee } & \multirow{2}{*}{ Total } \\
\hline & Present ${ }^{\star}$ & Absent & Present $^{* *}$ & Absent & \\
\hline Male & $51(6.3)$ & $141(17.3)$ & $60(7.4)$ & $136(16.7)$ & $388(47.7)$ \\
\hline Female & $40(4.9)$ & $165(20.3)$ & $45(5.5)$ & $175(21.5)$ & $425(52.3)$ \\
\hline Total & $91(11.2)$ & $306(37.6)$ & 105 (12.9) & $311(38.3)$ & $813(100)$ \\
\hline
\end{tabular}

Values are presented as number (\%). No significant difference with sex on the right side knee $\left({ }^{\star} P=0.09\right)$. Significant difference with sex on the left side knee ${ }^{*} P<0.01$.

Table 2. Association of presence of fabella with respect to age

\begin{tabular}{|c|c|c|c|c|c|}
\hline \multirow{2}{*}{ Age group (yr) } & \multicolumn{2}{|c|}{ Right knee } & \multicolumn{2}{|c|}{ Left knee } & \multirow{2}{*}{ Total } \\
\hline & Present ${ }^{*}$ & Absent & Present $^{* *}$ & Absent & \\
\hline $20-35$ & $4(0.5)$ & $58(7.1)$ & $8(1.0)$ & $62(7.6)$ & $132(16.2)$ \\
\hline $36-50$ & $15(1.8)$ & $59(7.3)$ & $9(1.1)$ & $69(8.5)$ & $152(18.7)$ \\
\hline $51-65$ & $26(3.2)$ & $63(7.7)$ & $31(3.8)$ & $62(7.6)$ & $182(22.4)$ \\
\hline $66-80$ & $27(3.3)$ & $59(7.3)$ & $26(3.2)$ & $60(7.4)$ & $172(21.2)$ \\
\hline$>80$ & $19(2.3)$ & $67(8.2)$ & $31(3.8)$ & $58(7.1)$ & $175(21.5)$ \\
\hline Total & $91(11.2)$ & $306(37.6)$ & 105 (12.9) & $311(38.3)$ & $813(100)$ \\
\hline
\end{tabular}

Values are presented as number (\%). Significant difference with age groups for right and left side knees respectively; ${ }^{\star} P<0.05$ (right side knee across age groups); ${ }^{*} P<0.01$ (left side knee across age groups). 
Table 3. Previous studies on the prevalence of fabella

\begin{tabular}{|c|c|c|c|c|c|}
\hline Study & Year & Ethnic group & Method & $\begin{array}{c}\text { No. of samples } \\
\text { (knees) }\end{array}$ & $\begin{array}{c}\text { Prevalence } \\
(\%)\end{array}$ \\
\hline Kaplan [29] & 1961 & Caucasian (America) & Cadaver & 135 & 8.7 \\
\hline Takebe et al. [32] & 1983 & Asian (Japan) & Radiography & NA & 33.4 \\
\hline Pritchett [33] & 1984 & Caucasian (America) & Radiography & 153 & 15 \\
\hline Yu et al. [13] & 1996 & Caucasian (America) & MRI & 100 & 19 \\
\hline Sarin et al. [6] & 1999 & Caucasian (America) & Radiography & 112 & 31.3 \\
\hline Minowa et al. [14] & 2004 & Asian (Japan) & Cadaver & 212 & 85.8 \\
\hline Kawashima et al. [5] & 2007 & Asian (Japan) & Cadaver & 150 & 66 \\
\hline Raheem et al. [34] & 2007 & Caucasian (Ireland) & Cadaver & 22 & 9.1 \\
\hline Silva et al. [11] & 2010 & Caucasian (Brazil) & Cadaver & 62 & 3.1 \\
\hline Phukubye and Oyedele [8] & 2011 & Caucasian and African & Cadaver & 102 & 23.5 \\
\hline Piyawinijwong et al. [30] & 2012 & Asian (Thailand) & Cadaver & 187 & 50.5 \\
\hline Zeng et al. [12] & 2012 & Asian (China) & Cadaver and Radiography & 61 & 86.9 \\
\hline Tabira et al. [31] & 2013 & Asian (Japan) & Cadaver & 102 & 68.6 \\
\hline Chew et al. [15] & 2014 & Asian (Singapore) & Radiography and MRI & 80 & 31.25 \\
\hline Egerci et al. [28] & 2017 & Caucasian (Turkey) & Radiography & 1000 & 22.8 \\
\hline Pop et al. [10] & 2018 & Caucasian (Romania) & Radiography and MRI & 431 & 16.93 \\
\hline Present study & 2021 & Asian (Oman) & Radiography; MRI & $813 ; 119$ & $24.1 ; 20.2$ \\
\hline
\end{tabular}

Adapted from Egerci et al. Folia Morphol (Warsz) 2017;76:478-83 [28]. MRI, magnetic resonance imaging; NA, not available.

systemic review study by Berthaume et al. (2019) [16], Hur et al. (2020) [20] estimated the overall prevalence rate of fabella reported from a total of 21,318 knees to be $20.2 \%$. They also estimated the average prevalence rate of the fabella in the Western population and Asian population to be $15.9 \%$ and $38.2 \%$, respectively [20].

In Omani subjects, the prevalence of fabella was found to be $24.1 \%$ in lateral radiographs and $20.1 \%$ in MRI films. This difference in prevalence could be due to the different inclusion criteria with respect to the age used in the two screening methods. As limited age group patients were evaluated in the MRI study, we considered $24.1 \%$ as the more accurate prevalence of fabella (based on radiography) in Omani patients. Our findings are nearly similar to prevalence rates reported from Turkey (22.8\%) [28]. Prevalence rate of fabella found in our study is within the ranges of $3.1 \%-31.3 \%$ that was reported in Western populations $[6,10,11,13,29]$ and lower than the average prevalence rate of $38.2 \%$ that was reported in other Asian populations. However, it is much lower than the prevalence rates reported in Far East Asian countries; China (86.9\%) and Japan (85.8\%) [14].

It has been demonstrated that genetic factors control the fabellar formation whereas environmental factors control its ossification [7]. Sox $9^{+} / \mathrm{Scx}^{+}$progenitors under the influence of transforming growth factor $\beta$ (TGF $\beta$ ), BMP2, and BMP4 have been found to participate in the development of fabella [7]. Variations in the prevalence rates among different ethnic groups further support the hypothesis of genetic factors influence on formation of fabella [2]. Since studying genetic factors was beyond the scope of this study, we cannot comment on this aspect based on our study findings. On the other hand, authors have demonstrated that the high prevalence rate of fabella in Far East countries could be due to certain functional or behavioral habits like better nutrition and daily praying practices where frequent use of kneeling and squatting movements may have promoted the fabellar development and ossification. This was further supported by phylogenetic theory, where the continuous mechanical stimuli over the posterior aspect of the lateral femoral condyle increases the ossification of the fabella [6]. The above mentioned behavioral habits are quite common in Omanis and may explain the presence of fabella in nearly $25 \%$ of our patient sample.

In the existing literature, sexual dimorphism in variations of the fabella is still debatable. A few studies have found no association between sex and the occurrence of fabella $[8,15$, 28]. However, evidence from the recent meta-analysis confirmed the sexual dimorphism in the prevalence of fabella with male dominance of $2.47 \%$ to $2.6 \%$ [2], which is similarly reflected in our study.

Development of fabella begins at 15 weeks of intrauterine life in the form of a cartilaginous nodule [35]. Although the exact age of the fabellar ossification is unknown, ossified fabellae were found at the age of as early as 12-14 years [36]. Various studies have quantified the association between the prevalence rates and age. Egerci et al. (2017) [28], Takebe et 
al. (1983) [32], and Tabira et al. (2013) [31] did not find differences in frequency of fabella among age groups. Contrary to these findings, Phukubye and Oyedele (2011) [8] and Kato et al. (2012) [37] found a higher prevalence of fabella in elderly individuals. Our study results concur with the recent meta-analysis which concluded that fabella prevalence rate increases with age [2].

Fabella can be present in the form of a cartilaginous or bony structure. Few cadaveric studies have found both cartilaginous and bony fabellae in their study subjects $[5,31]$. In one of these studies, the prevalence of cartilaginous fabellae was found to be $45 \%$ and in another study it was 32\% [31]. A cadaveric study described the structural details of the fabellae and categorized them into rigid and elastic structures. Around $36.3 \%$ of the identified fabellae were found to be elastic in structure. Moreover, the majority of the rigid fabella were osseous and a few were fibrotic in structure [12]. Among Asian population as reported by Chew et al. (2014) [15], all the identified fabellae were found to be bony structures with no cartilaginous fabellae identified following MRI screening. Similarly, in our present study all the observed fabellae were bony structures.

There are reports of pain in the posterolateral corner of the knee associated with the presence of fabella [27, 38]. Similarly, a high association between the presence of fabella and posterolateral knee pain has been reported by a population based study [10]. A house-to-house population study of different parts of Oman reported knee pain in 15\% of females and $18 \%$ of males [39]. Though the observed prevalence of fabella among Omanis is low, the results of the present study are of clinical importance while considering the differential diagnosis in patients with knee pain.

The main strength of the present study is that we could investigate both prevalence and characteristics of the fabella by studying both plain radiographs and MRI films. This study being from a single-center, the findings cannot be generalized outside of the study environment. Also since we included only those subjects who underwent radiological investigations, the possibility of underestimation of the prevalence due to the selection bias cannot be ruled out.

In conclusion, the reported prevalence of fabella in Omani subjects is $24.1 \%$. The present study is the first to document the prevalence of fabella in Omani population and will serve as a reference for future basic anatomical and orthopedic research in Oman. The reported baseline data of fabella is a clinically important consideration for the differential diag- nosis and thus aids in better management of patients with knee pain.

\section{ORCID}

Omar Darwish Al Matroushi:

https://orcid.org/0000-0001-8792-2638

Srinivasa Rao Sirasanagandla:

https://orcid.org/0000-0002-7261-1666

Ammar Al Shabibi:

https://orcid.org/0000-0003-4812-5091

Athari Al Obaidani:

https://orcid.org/0000-0002-8901-0592

Humoud Al Dhuhli:

https://orcid.org/0000-0003-2442-496X

Sanjay Jaju: https://orcid.org/0000-0002-9381-1528

Mohamed Al Mushaiqri:

https://orcid.org/0000-0002-2820-8968

\section{Author Contributions}

Conceptualization: ODAM, SRS, HAD, MAM. Data acquisition: ODAM, SRS, AAS, AAO. Data analysis or interpretation: SRS, HAD, SJ. Drafting of the manuscript: ODAM, SRS, AAS, MAM. Critical revision of the manuscript: SRS, HAD, SJ. Approval of the final version of the manuscript: all authors.

\section{Conflicts of Interest}

No potential conflict of interest relevant to this article was reported.

\section{References}

1. Duncan W, Dahm DL. Clinical anatomy of the fabella. Clin Anat 2003;16:448-9.

2. Berthaume MA, Bull AMJ. Human biological variation in sesamoid bone prevalence: the curious case of the fabella. J Anat 2020;236:228-42.

3. Theodorou SJ, Theodorou DJ, Resnick D. Painful stress fractures of the fabella in patients with total knee arthroplasty. AJR Am J Roentgenol 2005;185:1141-4.

4. Hauser NH, Hoechel S, Toranelli M, Klaws J, Müller-Gerbl M. Functional and structural details about the fabella: what the important stabilizer looks like in the central European population. Biomed Res Int 2015;2015:343728.

5. Kawashima T, Takeishi H, Yoshitomi S, Ito M, Sasaki H. Ana- 
tomical study of the fabella, fabellar complex and its clinical implications. Surg Radiol Anat 2007;29:611-6.

6. Sarin VK, Erickson GM, Giori NJ, Bergman AG, Carter DR. Coincident development of sesamoid bones and clues to their evolution. Anat Rec 1999;257:174-80.

7. Eyal S, Rubin S, Krief S, Levin L, Zelzer E. Common cellular origin and diverging developmental programs for different sesamoid bones. Development 2019;146:dev167452.

8. Phukubye $\mathrm{P}$, Oyedele $\mathrm{O}$. The incidence and structure of the fabella in a South African cadaver sample. Clin Anat 2011;24:84-90.

9. Zipple JT, Hammer RL, Loubert PV. Treatment of fabella syndrome with manual therapy: a case report. J Orthop Sports Phys Ther 2003;33:33-9.

10. Pop TS, Pop AM, Olah P, Trâmbiţaş C. Prevalence of the fabella and its association with pain in the posterolateral corner of the knee: a cross-sectional study in a Romanian population. Medicine (Baltimore) 2018;97:e13333.

11. Silva JG, Chagas CAA, Torres DFM, Servidio L, Vilela AC, Chagas WA. Morphological analyisis of the fabella in Brazilians. Int J Morphol 2010;28:105-10.

12. Zeng SX, Dong XL, Dang RS, Wu GS, Wang JF, Wang D, Huang HL, Guo XD. Anatomic study of fabella and its surrounding structures in a Chinese population. Surg Radiol Anat 2012;34:65-71.

13. Yu JS, Salonen DC, Hodler J, Haghighi P, Trudell D, Resnick D. Posterolateral aspect of the knee: improved MR imaging with a coronal oblique technique. Radiology 1996;198:199-204.

14. Minowa T, Murakami G, Kura H, Suzuki D, Han SH, Yamashita $T$. Does the fabella contribute to the reinforcement of the posterolateral corner of the knee by inducing the development of associated ligaments? J Orthop Sci 2004;9:59-65.

15. Chew CP, Lee KH, Koh JS, Howe TS. Incidence and radiological characteristics of fabellae in an Asian population. Singapore Med J 2014;55:198-201.

16. Berthaume MA, Di Federico E, Bull AMJ. Fabella prevalence rate increases over 150 years, and rates of other sesamoid bones remain constant: a systematic review. J Anat 2019;235:67-79.

17. Ghimire I, Maharjan S, Pokharel G, Subedi K. Evaluation of occurrence of sesamoid bones in the lower extremity radiographs. J Chitwan Med Coll 2017;7:11-4.

18. Kaneko K, Serizawa M, Genda N, Kikkawa F. [Einige Betrachtungen über die Fabella des Menschen]. Acta Anat Nippon 1966;42:85-8. German.

19. Robertson A, Jones SC, Paes R, Chakrabarty G. The fabella: a forgotten source of knee pain? Knee 2004;11:243-5.

20. Hur JW, Lee S, Jun JB. The prevalence of fabella and its association with the osteoarthritic severity of the knee in Korea. Clin Rheumatol 2020;39:3625-9.

21. Patel A, Singh R, Johnson B, Smith A. Compression neuropathy of the common peroneal nerve by the fabella. BMJ Case Rep 2013;2013:bcr2013202154.

22. Cesmebasi A, Spinner RJ, Smith J, Bannar SM, Finnoff JT. Role of sonography in the diagnosis and treatment of common peroneal neuropathy secondary to fabellae. J Ultrasound Med 2016;35:441-7.
23. Ando Y, Miyamoto Y, Tokimura F, Nakazawa T, Hamaji H, Kanetaka M, Koshiishi A, Hirabayashi K, Anamizu Y, Miyazaki T. A case report on a very rare variant of popliteal artery entrapment syndrome due to an enlarged fabella associated with severe knee osteoarthritis. J Orthop Sci 2017;22:164-8.

24. Franceschi F, Longo UG, Ruzzini L, Leonardi F, Rojas M, Gualdi G, Denaro V. Dislocation of an enlarged fabella as uncommon cause of knee pain: a case report. Knee 2007;14:330-2.

25. Kwee TC, Heggelman B, Gaasbeek R, Nix M. Fabella fractures after total knee arthroplasty with correction of valgus malalignment. Case Rep Orthop 2016;2016:4749871.

26. Zhou F, Zhang F, Deng G, Bi C, Wang J, Wang Q, Wang Q. Fabella fracture with radiological imaging: a case report. Trauma Case Rep 2017;12:19-23.

27. Kim T, Chung H, Lee H, Choi Y, Son JH. A case report and literature review on fabella syndrome after high tibial osteotomy. Medicine (Baltimore) 2018;97:e9585.

28. Egerci OF, Kose O, Turan A, Kilicaslan OF, Sekerci R, KelesCelik N. Prevalence and distribution of the fabella: a radiographic study in Turkish subjects. Folia Morphol (Warsz) 2017;76:478-83.

29. Kaplan EB. The fabellofibular and short lateral ligaments of the knee joint. J Bone Joint Surg Am 1961;43-A:169-79.

30. Piyawinijwong S, Sirisathira N, Sricharoenvej S. The fabella, fabellofibular and short lateral ligaments: an anatomical study in Thais cadavers. Siriraj Med J 2012;64(Suppl 1):S15-8.

31. Tabira Y, Saga T, Takahashi N, Watanabe K, Nakamura M, Yamaki K. Influence of a fabella in the gastrocnemius muscle on the common fibular nerve in Japanese subjects. Clin Anat 2013;26:893-902.

32. Takebe K, Kita K, Hirohata K. [Radiological and anatomical observation on fabella]. Orthop Surg 1983;34:1163-70. Japanese.

33. Pritchett JW. The incidence of fabellae in osteoarthrosis of the knee. J Bone Joint Surg Am 1984;66:1379-80.

34. Raheem O, Philpott J, Ryan W, O’Brien M. Anatomical variations in the anatomy of the posterolateral corner of the knee. Knee Surg Sports Traumatol Arthrosc 2007;15:895-900.

35. Jin ZW, Shibata S, Abe H, Jin Y, Li XW, Murakami G. A new insight into the fabella at knee: the foetal development and evolution. Folia Morphol (Warsz) 2017;76:87-93.

36. Ehara S. Potentially symptomatic fabella: MR imaging review. Jpn J Radiol 2014;32:1-5.

37. Kato Y, Oshida M, Ryu K, Horaguchi T, Seki M, Tokuhashi Y. The incidence and structure of the fabella in Japanese population. Anatomical study, radiographic study, and clinical cases. Paper presented at: ORS 2012 Annual Meeting; 2012; San Francisco, USA.

38. Merolla G, Dave AC, Paladini P, Campi F, Porcellini G. Ossifying tendinitis of the rotator cuff after arthroscopic excision of calcium deposits: report of two cases and literature review. J Orthop Traumatol 2015;16:67-73.

39. Pountain G. Musculoskeletal pain in Omanis, and the relationship to joint mobility and body mass index. Br J Rheumatol 1992;31:81-5. 